Originalveröffentlichung in: Faits de langues 27, Les langues chamito-sémitiques (afro-asiatiques), volume 2 (2006), S. 191-216

\title{
En route vers le copte. Notes sur l'évolution du démotique tardif
}

\author{
Joachim Friedrich Quack*
}

\section{INTRODUCTION}

\subsection{Le démotique}

Le démotique est une phase tardive de la langue égyptiennel. Il se situe entre le néo-égyptien et le copte. Aujourd'hui, il est bien reconnu comme étape réellement charnière entre ces deux entités ${ }^{2}$. On a l'habitude de diviser le démotique en trois phases: démotique archaïque, démotique moyen et démotique tardif 3 . Ces stades se trouvent commodément co-extensifs avec les périodes politiques : Époque tardive, Époque ptolémaïque et Époque romaine ce qui suffit à montrer que ces définitions sont un peu artificielles. En fait, il est utile de tenir compte de quelques faits essentiels : le développement de la langue parlée était assez continu - au moins, on serait obligé à montrer des changements décisifs avant de postuler une rupture. De plus, les étapes du démotique sont largement définies comme des phases paléographiques. Il manque encore une théorie nettement linguistique qui permettrait d'établir la date de composition d'un texte indépendamment de la date du manuscrit qui le transmet, et qui permettrait des jugements comme "écriture romaine, mais état de langue ptolémaïque".

Pour améliorer cette situation, je préfêre commencer avec la phase du démotique qui est déjà la plus nettement distinguée, à savoir le démotique tardif. Il a toujours été reconnu comme ayant des particularités qui le rapprochent du copte. Une analyse détaillée rendrait possible d'affirmer avec plus d'assurance quand certains changements de langue se sont produits et dans quelles conditions.

\footnotetext{
* Ruprecht-Karls-Universităt Heidelberg, Seminar für Ägyptologie; Courriel : Joachim_Friedrich.Quack@urz.uni-heidelberg.de

1 Parmi les ouvrages grammaticaux majeurs, on compte ceux de Spiegelberg (1925), Johnson (1976), Simpson (1996); of. aussi Lexa (1947-51).

${ }^{2}$ La theorie ancienne de Sethe (1925) et Grapow (1938), selon laquelle le démotique est un cul-de-sac, alors que le copte dérive directement du néo-égyptien, est dépassée, $c f$. par exemple Johnson (1976:298-301).

${ }_{3}$ Par exemple, Spiegelberg (1925:2sq)
} 


\subsection{Les sources du démotique tardif}

Malgré la période de temps considérable qui peut théoriquement être attribuée au démotique tardif, les sources sont assez peu nombreuses. La raison principale en est que les textes littéraires de l'époque romaine sont rarement des compositions vraiment nouvelles, mais plutôt des remaniements de versions plus anciennes (pour un bon exemple, voir Ryholt, 1999). Par conséquent, on ne trouve que rarement des constructions spécifiquement tardives, en général on reste dans l'usage du démotique classique. Quelques cas particuliers, où l'influence des constructions tardives est clair, seront cité dans le corps de cette étude.

Les textes non littéraires sont assez rares à l'époque romaine, et même parmi ce qui existe, assez peu de textes ont été rendus accessible de manière à permettre une analyse grammaticale.

Un groupe assez important sont les ostraca de Narmouthis (Medinet Madi) ${ }^{4}$. Ils semblent dater de la deuxième moitié du 2ème siècle ap. J.-C. Malheureusement, les cas instructifs dans ce qui est déjà publié à ce sujet ne sont pas suffisants pour établir un système. On pourrait plutôt expliquer leurs usages significatifs une fois que les particularités du démotique tardif auront été définis d'après d'autres sources.

Comme source privilégiée, il reste encore les papyrus magiques de l'époque romaine, spécialement le grand papyrus magique de Londres et Leyde, écrit au 3ème siècle après J.-C. ${ }^{5}$. En fait, j'ai préféré travailler presque exclusivement sur ce papyrus pour atteindre plus d'homogénéité dans les résultats. Pour les autres papyrus magiques de cette époque, il faut dire que, soit ils sont encore largement dans un stade moins développé de la langue ${ }^{6}$, soit ils sont mal conservés?. Même pour le papyrus de Londres et Leyde, la question de l'homogénéité pose un problème bien plus grave qu'il n'est normalement supposé. Il faut tenir compte de la nature du document qui n'est pas une composition uniforme. On a plutôt une compilation de recettes à usage personnel, provenant de sources différentes et probablement variables dans leur usage de la langue. Un des objectifs de ma recherche est d'ailleurs de montrer que certaines sections du papyrus ne sont pas à considérer comme du démotique tardif, mais comme des vestiges traditionnels avec, au maximum, quelques remaniements récents. Les sections les plus nettement archaïques sont pMag. LL. $6,11-7,5$ et $11,1-11,26$, et peut-être 15,1-23;9,1-10,22 semble aussi un peu plus ancienne que beaucoup d'autres sections.

4 Bresciani, Pemigotti \& Betrò (1983); Gallo (1997). La traduction de ces ostraca pose encore de nombreuses difficuités.

5 Édition Griffith \& Thompson (1904-09); pour des détails paléographiques, les publications de Hess (1892) et Leemanns (1839) peuvent encore être utiles. L'étude grammaticale de Ort-Geuthnet (1936) est aujourdhui de peu de secours; mais le texte a été utilisé comme une des sources principales dans Johnson (1976).

${ }^{6}$ Ainsi le papyrus Louvre E 3229 (édition Johnson, 1977), dont seules les sections mal conservées $1,1-3,1$ et le verso semblent être en démotique vraiment tardif, alors que les autres sections sont dans une langue plus traditionnelle.

${ }_{7}^{7}$ Ainsi le papyrus Leyde I 384 vs., édition Johnson (1975). 


\subsection{Méthode}

Une description complète de l'usage de la langue du papyrus serait hors propos. Il faut se concentrer sur les faits de langue qui sont le plus susceptibles de montrer l'apparition des innovations, surtout dans le systeme verbal. Pour mieux apprécier le caractère particulier du démotique tardif, les usages normaux du démotique (archaïque et ptolémaïque) et du copte pour les constructions en question seront indiqués. Ensuite, la confrontation avec les attestations actuelles du corpus permettra de cerner la position du démotique tardif par rapport aux phases voisines de la langue.

\section{LE FUTUR I}

La forme nouvelle la plus marquante qui fait son apparition en démotique est le futur I. En copte, il est formé avec les pronoms proclitiques du présent I ou un substantif, suivi de la marque temporelle $n a$, ce qui donne fnasōtm "il écoutera". Cette forme sert largement pour le futur objectif alors que le futur III efesōtm a généralement des fonctions modales, comme "il doit écouter" (voir Layton, 2000:239sq et 263-267). Étymologiquement, le futur III tire son origine de la construction $i w=f m n^{c} i r$ sčm "il est en train d'écouter", attesté en néo-égyptien, mais sans grammaticalisation (Černý \& Groll 1984:339-341). En démotique, il n'est pas utilisé avant la période romaine. Un exemple qui n'est pas encore grammaticalisé est $l: i r=k i n . n^{c} . k n k t . k$ "quand tu es sur le point de dormir" (pLouvre E 3229, 5, 21).

La forme réelle du futur I est assez rare. Dans le papyrus magique de Londres et Leyde, l'orthographe de la marque temporelle est toujours non étymologique. La construction est attestée dans une phrase principale, comme tw $=y$ n $3 e$ h.sy. $F k$ "je vais te louer" (pMag. LL. 20, 19; sim. 20, 20), dans une phrase relative avec le mot de référence comme sujet, comme p\} ner nll n\}e lrip $\{y=l$ śni $n$ p 3 hrw "le dieu qui fera mon enquête aujourd'hui" (pMag. LL. 3, l, sim. 3, 4), mais aussi

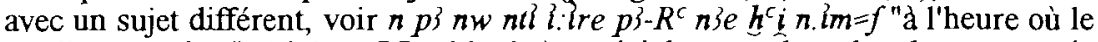
soleil apparaîtra" (pMag. LL. 29, 2s.); spécialement dans la phrase coupée $m t w=k$ nil nje šni "c'est toi qui demanderas" (pMag. LL. 23, 21); et dans la

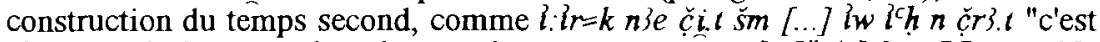
alors que la lune est dans le scorpion que tu mettras [...]" (pMag. LL. vs. 28, ls.).

Il y a toutefois des restrictions importantes à noter. La construction du futur I est encore rare, alors que le futur III est largement utilisé comme la forme normale du futur. Bien plus, le futur I n'est pas encore attesté avec une négation. Un cas instructif est pMag. LL. 8, 14s. : on a $t$ ' mt.t n3e hpr "la chose se produira", mais $b n-l w=s r h p r$ "elle ne se produira pas" avec la négation du futur III 8 .

${ }^{8}$ I faut noter que la présence de la préposition $r$ dans cette construction, bien que justifiée étymologiquement, n'est pas d'usage en démotique, ni requise par la prononciation (copte $n n e f s o ̈ t m$ sans trace de $e$ après $n n e f$ ). 
En dehors du grand papyrus magique, on trouve quelques attestations isolées du futur III dans des textes d'époque romaine. Il y a ceux qui conservent encore l'écriture originale, comme $t w=y \quad n^{c} i \breve{c y} s t$ "je le donnerai" (Late Demotic Gardening Agreement B 19, d'après Parker, 1940:99), et ceux qui se présentent dans une forme non étymologique comme $t=l n\}$.w $s m\} \quad r$ p $t w$ "je bénirai la montagne" (pWien D 6920 rt. $x+2,7)^{9}$. En résumé, on observe une forme naissante, qui commence à trouver sa place dans le système grammatical pendant l'époque romaine, pour devenir tout à fait normale en copte.

\section{LA RESTRUCTURATION DU CONJONCTIF}

La forme démotique normale du conjonctif est le préfix mtw suivi d'un suffixe ou d'un substantif. En copte, la formation a changé, et elle est variable selon le dialecte. En sahidique, on trouve nte devant un substantif, ce qui correspond encore à l'état ancien, mais pour les pronoms personnels, le conjonctif a été remodelé; une analyse synchronique pourrait le décrire comme un $n$ suivi par les pronoms proclitiques du présent I (sauf pour la première personne du singulier où on a nta alors que le présent I utilise le préfix $t i$ ). En akhmimique, les préfixes sont même totalement identiques à ceux du présent I et la seule distinction entre les deux formes est que le conjonctif ne tolère que l'infinitif comme prédicat, et peut ajouter un complément d'objet direct au verbe sans médiateur.

En bohairique, au contraire, on a la base nte pour toutes les personnes, donc une morphologie plus conservative - avec toutefois une particularité notable. Pour la troisième personne du pluriel, on trouve les deux formes $n t u$ et $n s e$ qui gardent cependant une nette distinction d'usage (Stern, 1880:273f. § 441),

Comme le papyrus magique de Londres et Leyde provient de Haute-Egypte, on s'attendrait à une situation qui annoncerait l'état sahidique. En réalité, la forme largement majoritaire est la forme traditionnelle, avec le morphème $m t w$ (voir la liste dans Johnson, 1976:283). Mais certaines attestations pour la deuxième personne du singulier et pour la troisième du pluriel montrent une orthographe nouvelle, celle des préfixes du présent I augmenté de l'élément $n$. Les formes utilisées sont $n \cdot l:\{r=k$ (pour $n k$ ) et $n$.st (pour $n s e$ ). Ainsi on a $i m$ $n .\{. l r=k$ wnm "viens et mange !" pMag. LL. vs. 33, 3 (autres exemples pMag.

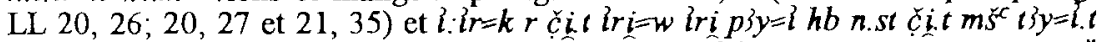
lp.t "tu les feras exécuter mon affaire et ils doivent faire progresser mon ceuvre" pMag. LL 2, 9. Peut-être y a-t-il aussi une attestation de cette morphologie nouvelle dans un ostracon de Medinet Madi, où l'on peut comprendre kty $n$ 3l. $i r=k$ šms "tourne et sers" (oMed. M. 65, 7).

Encore une fois, on assiste à la naissance d'une morphologie nouvelle. Les passages où elle apparaît peuvent être considérés comme les plus récents du manuscrit. Cette phase transitoire dans laquelle la morphologie du conjonctif est profondément remodelée pourrait expliquer une forme aberrante. En pMag. LL

${ }^{9}$ Hoffmann (1996a:173f. n. 53). Peut-être aussi oMedinet Madi 18, 8s., mais la lecture du verbe est peu sure (peut-être $i b[h]$ "oublier"). 
vs. 33,3 , on a $\left.i m n \cdot l: l_{r}=k n\right\} e w n m$, ce qu'il serait logique de traduire comme "viens et mange", avec la construction normale du conjonctif pour continuer un impératif. Toutefois, la morphologie avec l'incorporation de $n \dot{i} e$ milite contre une analyse comme un conjonctif normal. On pourrait à la rigueur essayer de l'analyser comme une question "Viens! Est-ce que tu vas manger ?". Mais le parallèle au conjonctif cité plus baut invite à regarder aussi ce passage comme un conjonctif. Dans une phase transitoire, pendant que la morphologie du conjonctif se rapproche du présent I, on aurait pu essayer de l'appliquer aussi au futur I qui, somme toute, est dérivé du présent I.

\section{LA MORPHOLOGIE DU CONDTTIONNEL}

La morphologie du conditionnel démotique traditionnel est caractérisée par une nette bipartition. Devant un substantif, on a le préfixe $i n-l w$ en démotique archaïque, écrit normalement plus tard $i n-n\} . w$. Devant un suffixe, par contre, on ne trouve la forme $i n-l w$ que dans les textes saïtiques et perses (voir Quack, $2000 a$ ). Plus tard, la première partie se perd et on obtient la forme $i w=f s c c m$. En copte, cette forme peu reconnaissable est renforcée par l'élément san, qui est presque obligatoire pour la forme affirmative (sauf dans le dialecte mésokémique et quelques attestations isolées; voir Shisha-Halevy, 1974, et la littérature citée dans Schenke, 1981:35 n. 60) mais optionnel pour la forme négative. De plus, la force de systématisation a mené à une forme analogique pour la morphologie avec des substantifs. Là, on utilise le préfixe ere, ce qui correspond à la situation du futur III, du présent II et de la forme circonstancielle du présent I où un $e$ avec un suffixe est toujours en relation paradigmatique avec ere devant un substantif.

La situation en démotique tardif montre une nette progression vers le copte. La forme ancienne du préfixe devant les substantifs ne se trouve qu'une fois, dans $l_{n-n\} . w}{ }^{w} n h$ h. $t h p r$ "s'il se produit un retard" (pMag. LL.6, 21) ${ }^{10}$, dans une section qui est très vraisemblablement ancienne. Ailleurs, la forme moderne, avec lifire ou rilre (aussi r:iri) comme notation du préfixe, est généralisée (pMag. LL. 7,$27 ; 12,4 ; 12,6 ; 12,11 ; 18,26 ; 21,13 ; 21,14 ; 25$, $30 ; 29,16 ;$ vs. 6,$7 ; 7,3$. 6f:; 26, 4) ${ }^{11}$; elle se trouve aussi dans le pLouvre $\mathrm{E}$ 32292 , 28, et probablement dans l'ostracon Medinet Madi 37, 1512. Devant les suffixes, la forme brève à préfixe $l_{w}$ est normale. Un seul exemple montre la situation normale du copte, a savoir : $\left.l: l r=k s^{c}-n\right\} e w w$ "si tu les envoies" (pMag. LL. 3, 29f.) où $\check{s}^{c}-n 3 e$ est visiblement une autre notation de $s a n$. Il se peut qu'il soit étymologiquement à rattacher au verbe $\check{s}^{\zeta C}$ "commencer" (ainsi Sethe, 1925:296 n. 1), mais c'est peu sûr. À noter aussi que la périphrase $i w=f$ hpr "s'il se passe [... $]^{\text {"' }}$ suivie du conjonctif ou du circonstanciel est bien attestée.

10 Johnson (1976:240) suppose que la forme simple en in $(6,14$ et 6,37$)$ est une forme du conditionnel, mais il s'agit plutôt de la particule interrogative.

11 La forme $l_{w}$ en 6,34 , donnée comme conditionnel dans Johnson (1976:234) est plutôt la forme circonstancielle du présent I.

12 Gallo 1997, p. 124 n. 35, propose, par contre, d'y voir un temps second. 
Elle se rencontre même une fois (pMag. LL. 3, 19) sous la forme abrégée îwhpr, qui correspond au esōpe copte.

Les formes négatives présentent quelques phénomènes remarquables. D'abord, avec des suffixes, ils utilisent le préfixe r:lri de préférence $(5,31 ; 14$, $4 ; 19,5 ; 28,8)$, et seulement occasionnellement la forme simple iw $(17,16)$. La raison phonétique pour cette graphie n'est pas encore claire mais selon les conventions du manuscrit, cela semble indiquer une prononciation *er(e) plutôt que $e$. D'un intérêt tout particulier est aussi la seule attestation de la forme

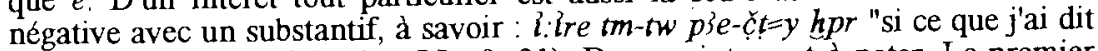
ne se produisait pas" (pMag. LL. 9, 31). Deux points sont à noter. Le premier est la position de la négation avant le sujet, et non pas après comme il est de norme avec un suffixe. En fait, la position à la première place de la négation, qui est de règle en copte, est optionnelle en démotique ${ }^{13}$. Le deuxième point est la forme étrange $t m-t w$ au lieu de $t m$ simple. Jusqu'ici, on a proposé de l'émender (comme Johnson, 1976:260-262). En fait, elle devrait être correcte, puisqu'il y a un phénomène parallèle en copte, où une forme exceptionnelle ertmte pour le conditionnel nié est attestée cinq fois dans le codex III de Nag Harnmadi (voir Emmel, 1984:35).

Somme toute, les formes du conditionnel montrent une nette progression vers l'état copte dans le démotique tardif, mais pas encore tout à fait les mêmes normes.

\section{LA DESTINEE DE $s c ̧ m=f$}

\subsection{Généralités}

Pendant la plus grande partie de l'existence de la langue égyptienne, la formation dite $s c \breve{m}=f$ est en vigueur, ou pour le dire de façon plus abstraite, la morphologie verbale est synthétique. En copte, elle est toujours analytique, avec une réduction des formes verbales à l'infinitif et au qualitatif. Toute la flexion se produit à l'aide de préfixes, souvent dérivés des constructions périphrastiques utilisant des verbes auxiliaires, en l'occurrence $i r i$ "faire". Bien sûr, le processus qui a mené à l'extinction de la conjugaison synthétique a été lent. En néoégyptien déjà, la conjugaison $s c ̧ m=f$ est réduite essentiellement à deux fonctions : le passé et le prospectif (Winand, 1992:192-198 et 209-258), et elle n'est plus possible pour des verbes ayant plus que trois consonnes radicales. Pour le présent et la plupart des usages du futur, on utilise des formes périphrastiques avec des prépositions.

La restriction quant au nombre des consonnes radicales est préservée en démotique, mais par ailleurs, $s c \breve{c}=f$ gagne du terrain, et cela pour deux raisons : l'une est la grammaticalisation de l'aoriste comme forme morphologique distincte. Il est construit par la combinaison du préfixe $h r$ avec sçm=f. L'autre

13 Pour la variation dans un seul manuscrit, cf. le Codex Hermopolis 7, 15 (position de tm avant le substantif) contre le Codex Hermopolis 6, 11 (position de $t m$ après le substantif). 
est un changement majeur dans la structure du récit au passé. En néo-égyptien, la forme séquentielle $\mathfrak{l} w=f h r$ sçm continue les formes narratives initiales. Dans la réalité des textes, c'est la construction dominante des passages narratifs. Mais elle disparaît pendant la 3ème Période Intermédiaire ${ }^{14}$, et en démotique, une chaîne de formes $s \grave{c} m=f$ est normale, en récit aussi bien qu'en discours, pour le passé.

Pour le démotique tardif, on s'attendrait à un mouvement vers le copte. En fait, la situation est passablement compliquée et il est approprié d'analyser chaque fonction de $s c ̧ m=f$ à part.

\subsection{Le prospectif autonome}

Je commence avec une forme qui n'est plus vivante à cette époque, à savoir le prospectif autonome, non enchâssé dans la construction causative. Cet emploi est encore possible en démotique archaïque et ptolémaïque dans certaines constructions bien définies, spécialement pour continuer un impératif, un optatif ou un futur III injonctif ${ }^{15}$. Ainsi, il est en concurrence avec le conjonctif démotique, et aussi avec la forme tarefsōtm du copte, ce qui aide à expliquer la rareté du prospectif dans cet usage, puis sa disparition. Dans le papyrus magique, on trouve encore trois ou quatre exemples. Deux d'entre eux apparaissent dans le même charme magique qui montre par plusieurs particularités qu'il n'est pas à considérer comme démotique tardif mais comme une composition plus ancienne : In $l: i r=k$ (r) $l y i k b=y \quad k=k$ "est-ce que tu vas venir, de sorte que je t'envoie ?" (pMag. LL. 6,31 ); my $c_{S=w}^{\prime} n=y{ }^{3} w^{c} n c r h b=y$ $s$ "qu'on appelle pour moi un dieu, afin que je l'envoie !" (pMag. LL. 6, 34). Une ou deux autres occurrences apparaissent dans le deuxième charme magique qui peut être regardé comme ayant une origine assez ancienne -- en fait, dans la souscription il est attribué à un roi dont seulement le $\breve{s}$ final est préservé, mais cela suffit pour réduire le cercle des possibilités à Darius, Xerxés, Artaxerxès et Khababach, tous de la période archaique du démotique. Cette attribution, bien sûr, peut être pseudépigraphique, mais il est quand même notable qu'elle se trouve justement dans un charme dont le caractère linguistique montre des

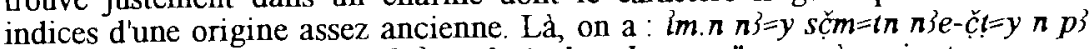
$h r w n h m=t n m$ nene $n b \stackrel{c}{\zeta} n b l h y n b t 3 n b m h r w$ pn "venez à moi, et que vous écoutiez ce que j'ai dit aujourd'hui et que vous (me) ${ }^{16}$ sauviez dans chaque détresse, chaque mal et chaque mauvaise affaire en ce jour" (pMag. LL. 11, 16s.). Peut-être peut-on aussi inclure $[m y(?) s c ̧ m]=k h r w=y n h m=k$ I $m w$ ihy $n b$ t) "que tu [écoutes] ma voix et que tu me sauves de chaque chose mauvaise"

14 Pour la question de la date exacte, voir Quack (2001:170-172).

is Il se trouve normalement là où le sujet change, mais il y en a aussi des exemples quand le sujet reste le même, voir Ankhchechonqi 6, 7. Les détails ont été peu étudiés jusqu'ici.

$16 \mathrm{La}$ non-écriture du pronom enclitique de la lère personne sG est typique pour le dérnotique archaique, surtout dans le papyrus Rylands IX (an 9 de Darius I), voir Vittmann (1998:234). 
(pMag. LL. 11, 3) mais là, le début de la phrase est perdu, de sorte qu'on ne peut pas juger s'il y avait une forme injonctive d'introduction ou non. Le seul exemple certain d'un prospectif tout a fait autonome est $c_{\text {hhe }} p 3 y=$ in by "que votre âme vive" (pMag. LL. 10,7), et il est modelé d'après des formules religieuses traditionnelles - en fait les formules religieuses sont les seuls cas en démotique où un prospectif autonome est encore d'usage fréquent. Archaïque aussi est $s \breve{c} m=k h r w[=y]$ "que tu entendes [ma] voix" (pLouvre E 3229, 2, 18).

\subsection{Le prospectif dans la construction causative}

Beaucoup plus fréquent est le prospectif dans la construction causative, dépendant du verbe $\breve{c i t}$ "donner, causer". En fait, pour la plus grande part de l'histoire du démotique, cette formation est d'un usage tout à fait libre. En copte, par contre, elle est lexicalisée et restreinte à un groupe de verbes figés. Mon intention est de montrer que l'état du démotique tardif dans ce domaine est déjà largement celui du copte ${ }^{17}$. Pour les verbes qui n'ont pas de causatif lexicalisé, on utilise la circonlocution $\check{c} i t$ tri $=f s c m$. Pour l'illustrer, je donne un inventaire des verbes utilisés avec un $s \breve{c r m}=f$ après une forme du verbe $\breve{c} \grave{i}$, toujours en indiquant comme élément de comparaison le verbe causatif figé du copte :

- çi.t iwi "envoyer", copte tawo; pMag. LL. 3, 18;4, 22; 6, 33; 7, 4; 8, 2. 7; 20, 31. 32 (bis); 29, 3; 29, 7;29, 11;29, 11;29, 15;29, 19; vs. 2, 15; pLeyde I 384 vs. *I, 3 .

- ci.t (3)y "glorifier", copte tayo; pMag. LL. 7, 11.

- ç.t. (c)lk "guérir", copte talco; pMag. LL. 19, 38; vs. 6, 1; vs. 10, 10f; vs. 20,1 .

$2 ; 29,21$; vs. 33,4 .

- čit $w^{c} b$ "punfier", copte $t b b o$; pMag. LL. 3, 17, 5, 3.

- či.t $w \breve{c}\}$ "faire prospérer", copte tujo; pMag. LL. 1, 8; 1, 26; 2, 11; 9, 12; 9, 14. 9, $30 ; 18,19$ (bis); 18. 30; 19, 15 .

- çitt-wn lr. $=$ "instruiren", copte tunyat $=$, pMag. LL. 1, 11. 18; 5, 22; 29, 22; vs. 22, 11. 16. 19; vs. $24,8.12$.

- čl.t wnm "alimenter, faire manger", copte tmmo, 2, 29; 3, 1; 13, 12;13, 22;18, $31 ; 21,40 ; 24,16$

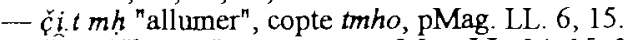

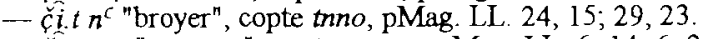

- çi.t nw "montrer", copte tanwo, pMag. LL. 6, 14; 6, 29 (bis); 7, 23. 31; 27, 2; 17, 8.

- çi.t hmsi "placer (assis)", copte thmso, pMag. LL. 2, 28; 10, 15; 16, 24; 17, 22; 21,40 ; vs. 8,2 .

- द́i.t hpr "créer, acquérir", copte jpo, pMag. LL. 2, 27; 3, 10; 5, 14; 5,18 u. 7, 14; $17,6.31 ; 5,18$ u. 7,$14 ; 17,6$ f.. $32 ; 8,9 ; 9,14 ; 11,12 ; 11,20 ; 11,25 ; 13$, $21 ; 14,11 ; 17,26$.

- çi.t swr "donner à boire", copte tso, pMag. LL. 2, 29 u. 3, 1; 13, 11f; 15,1; 15, 7; 15,$23 ; 18,31 ; 19,10 ; 19,20 f ; 19,40 ; 20,33 ; 21,17 ; 21,40 ; 24,14 ; 24,33$; vs. 5,2 ; vs. 32,9 .

17 Déja dans les ostraca de Medinet Madi, on trouve des attestations de la construction périphrastique, voir oMM 50, 7f:; 82, 6 . 
- či.t st "renvoyer", copte tsto, pMag. LL. 17, 20.

- $\breve{c}$ i.t sčr "placer couché", copte jto, pMag. LL. 3, 12;21,40; vs. 2, 16.

- çi.t śm "envoyer" 18 , copte jol9, pMag. LL. 2,$12 ; 18,30 ; 3,28 ; 3,31 ; 9,21 ; 18$, $15.18 ; 21,12 ; 28,6 ; 28,15$; vs. 8,2 ; vs. 8,3 ; vs. 28,1 .

- či.t twn $=f s$ "éveiller", copte tunos, pMag. LL. 19, 31

- $\breve{c} . t$ kte "tourner", copte Txтo, pMag. LL. 9, 31; 10, 13; vs. 4, 17; pLeyde 384 vs. *I, 4.

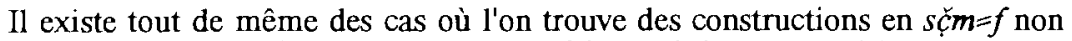
périphrastique, là où il n'y pas de causatif lexicalisé en copte. Un examen en détail s'avère fructueux. On a par exemple les cas suivants :

- iri "faire", pMag. LL. 3, 1; 7, 23; 10, 8; 12, 14; 15, 14; 15, 17; 15, 19f; 17,$19 ; 21,38$; vs. 5,4 f.; vs. 12,9 . Comme c'est justement le verbe qui sert aussi d'auxiliaire, on peut comprendre qu'il est censé ne pas avoir forcément besoin d'une forme périphrastique. En fait, de rares cas de la construction périphrastique (voir plus bas) illustrent comment ce verbe a été soumis à la norme, peut-être un peu plus tard que le reste des verbes.

_- $c_{S}$ "crier", pMag. LL. 6, 34. C'est un passage en langue plus traditionnelle. En démotique tardif normal, on trouve la forme périphrastique (pMag. LL. 2, 1).

—wšt "vénérer", pMag. LL. 10, 25f. $=27,4 ; 27,20$. Ces deux exemples (l'un est un doublet) proviennent aussi de passages qui donnent l'impression d'être plus traditionnels.

- phr "encercler, enchanter", pMag. LL. 18, 9; 29, 11. Le verbe n'est plus attesté en copte, ni à la forme simple, ni comme causatif. D'ailleurs, la construction simple est en concurrence avec la construction périphrastique qui est attestée en pMag. LL. 3, 33.

- $m 33$ "voir", pMag. LL. $5,19=7,15 ; 5,20=7,15 ; 7,16 ; 11,2 ; 29$, 19. De nouveau, c'est un verbe qui n'est plus attesté en copte; en fait, même en démotique, il est tombé en désuétude et doit être considéré comme un archaïsme. On peut le prouver par une formule attestée dans plusieurs versions. Dans un cas (pMag. LL. 17, 8; parallèle à 7, 16), on a remplacé le verbe $m 33$ par $\pi w$, le mot normal pour "voir" en démotique.

- $m \check{s}^{c}$ "aller", pMag. LL. 2, 9. Pour le moment, il n'existe pas d'explication évidente.

- či.t nfr "embellir", pMag. LL. 20, 24. Il existe encore de rares attestations du verbe-adjectif nefr en copte, mais il est tombé normalement en désuétude.

- lbe "être excité", pMag. LL. vs. 29, 1. Deux attestations emploient la construction périphrastique (pMag. LL. 13,17; vs. 32, 1). Il ne faut pas exclure qu'on a ici aussi un passage plus traditionnel; aucune des autres constructions de cette brève section ne permet d'établir un jugement sur cette question.

- htp "se reposer", pMag. LL. 15, 30. Il se peut qu'un causatif lexicalisé originalement présent ait été abandonné en copte, parce qu'il devenait phonétiquement trop similaire à thpo (métathèse de çilt-ph).

18 Dans quelques cas, le mot est écrit non étymologiquement $\check{c} 3 i$.

${ }_{19} \mathrm{Y}$ inclus les formes de $\breve{c} i . t s m^{\text {"faire }}$ partirn", jne-, $m a=$ en copte 
- sčm "écouter", pMag. LL. $5,20=7,15 ; 7,16 ; 17,8 ; 18,1$. On peut se demander si la forme copte tsamo "enseigner" (prise jusqu'ici pour une variante phonétique de $t s a b o$ ) n'est pas à dériver d'un *tsatmo, ce qui donnerait un causatif lexicalisé pour ce verbe.

- ک̌w "être sec", pMag. LL. 21, 35; vs. 19, 2. En copte, on trouve ऊ̌wo qui semble bien être un causatif lexicalisé $\left(* t s_{w o}\right)$, mais il signifie "vider".

- ct "parler", 6,$32 ; 7,29 ; 8,9 ; 14,15 ; 29,11$. Il faut noter que tous ces exemples apparaissent dans la construction optative $m y \quad \check{c} t=f$ "qu'il dise". Ailleurs, la construction périphrastique est attestée pour le même verbe, y inclus d'autres exemples de l'optatif (voir plus bas).

Sont à discuter particulièrement les quelques cas qui donnent l'impression que les deux constructions sont possibles pour le même verbe. Mais dans bien des cas, il y a de nettes différences entre les deux expressions. Pour le verbe ly $i / l w i$ "venir", la construction directe $c ̧ i . l$ lwi "envoyer" désigne toujours le mouvement vers l'extérieur, quelques fois même avec un sens métaphorique "annoncer". Au contraire, les deux attestations de la formule périphrastique či.t iri $=w$ lyl (pMag. LL. 3, 23; 3, 31) "les faire entrer" expriment un mouvement vers l'intérieur (la préposition $r \underline{h n}$ "vers l'intérieur" est présente dans les deux cas)

Encore plus significatif est l'usage pour wn ir.t "ouvrir l'œil". Il existe une expression idiomatique $\breve{c} i t w n l r . t=q u i$ ne signifie plus "faire ouvrir l'cil", mais "instruire". Cette expression idiomatique (qui donne tunyat = en copte) est toujours utilisée dans la forme simple, alors que pour le sens littéral "faire que quelqu'un ouvre son œil", on utilise toujours la construction périphrastique (pMag. LL. 2, 15;3, 15; 7, 27;14, 5; 16,25f. 6, 6;17, 12;14, 24;18, 6; 27, 16).

On peut aussi montrer une distinction de sens pour le verbe ${ }^{c} h^{c}$ : on a le causatif lexicalisé dans le sens de "ériger, étendre, atteindre". Mais my $l r i=f^{c} h^{c} r$ $r t . f f$ (pMag. LL. 3, 2) signifie "qu'il se mette debout"; alors que *my ${ }^{*} \widehat{h}^{c}=f r r t$ $\ell f$ serait "érige-le debout".

Pour le verbe $h p r$, le causatif lexicalisé est usuel, mais il existe des attestations périphrastiques, par exemple : $m y$ ire $m t . t-m \zeta^{c} h p r$ "que la vérité se produise" (pMag. LL. 9, 28; sim. 9, 30f; 10,3; 16, 2lf). Tous se trouvent dans la construction optative, ce qui aiderait à les comprendre. La lexicalisation de la construction causative concerne surtout la forme $\check{c} i \mathrm{i} t$-sçm. Elle est moins évidente dans les cas où on a l'impératif du verbe $c \grave{i} . t$, qui est $m y$. Cette forme de l'impératif est aussi à la base de l'optatif, et la discission est ouverte concernant $m y s c \breve{c} m=f$ qui peut se comprendre comme causatif "fais qu'il écoute" ou comme simple optatif "qu'il écoute". Dans la plupart des cas, la construction de l'optatif suit la tendance générale pour les causatifs lexicalisés; pour cette raison, je l'ai

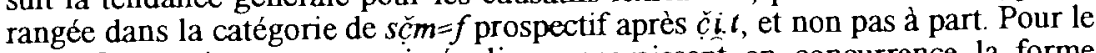
verbe $h p r$, qui est un peu irrégulier, apparaissent en concurrence la forme simple my hpr (pMag. LL. 9, 14;14, 11; 17, 26), la forme périphrastique, et même la construction $m y$ çit. hop $t 3 m^{c} . t$ (pMag. LL. 2, 27) qui possède 
l'élément causatif en double (Depuydt, 1998:234; pour les causatives à double élément, voir plus bas).

Pour $n w$ "voir, regarder", à côté du causatif lexicalisé, il y a aussi la construction périphrastique (pMag. LL. 17, 15; 17, 29). Pour ce verbe, il faut dire que le causatif lexicalisé est très rare en copte, alors que l'utilisation de la construction périphrastique est normale. Il n'est pas surprenant qu'elle soit déjà possible en le démotique tardif.

Pour lby, la forme périphrastique attestée en 13,17 et vs. 32, 1 doit être considérée comme normale. La construction simple (pMag. LL. vs. 29, 1) est, comme il a déjà été dit, surprenante, étant donné qu'il n'existe pas de causatif lexicalisé de ce verbe en copte.

De même, pour $p h r$, la construction périphrastique (pMag. LL. 3, 33) est celle à laquelle on s'attendrait, étant donné qu'il n'existe pas de causatif lexicalisé en copte. Les constructions périphrastiques de ç̣! (pMag. LL. 3, 3; 3, 4; 9, 22. 27; $10,1)$ s'expliquent de la même manière.

Le cas de sčr en construction périphrastique (pMag. LL. 14, 19) reste énigmatique, étant donné surtout que le verbe n'est plus attesté en dehors du causatif lexicalisé en copte.

Finalement, le verbe $i r i$ "faire" peut utiliser la construction périphrastique, mais plutôt rarement (2, 9 (bis); 14, 14f; vs. 12, 8; pLouvre E 3229, 2, 24). Une explication possible est donnée plus haut.

La lexicalisation de l'ancienne construction causative a conduit à un développement logique : dans la mesure où l'on considère un mot comme talo

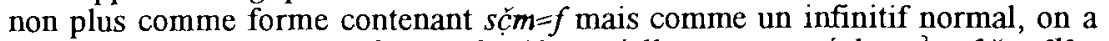
besoin d'un qualitatif du même verbe. Il est réellement attesté dans $i_{w}=f \quad c i l t-q y_{l}$ (pMag. LL vs. 33, 1), à comparer avec le copte talait. Une forme analogue (bien que non dérivée d'un causatif lexicalisé) est $\measuredangle 3$ i-r3.t (pMag. LL 14, 21); var. $\varnothing 3$ ir.y (pMag. LL 16,11; vs. $31,3 \mathrm{f}$ ), a comparer avec le copte jerait, du verbe *çi.t h.t $r$ "allumer", noté inétymologiquement comme $z_{3 i-r}$ '. Il semble que de telles formations du qualitatif sont modelées sur l'analogie avec le verbe $h k o$ "avoir faim", qualitatif hkoit ou hkait, un des rares cas d'infinitif d'un verbe non causatif se terminant en $o^{20}$. Une telle formation montre bien que le processus de lexicalisation des causatifs est complet en démotique tardif.

Un autre indice va dans le même sens. En démotique, comme en copte, il existe une règle stipulant qu'un complément d'objet direct dans un temps duratif doit être introduit par un médiateur (voir plus bas). Mais les constructions

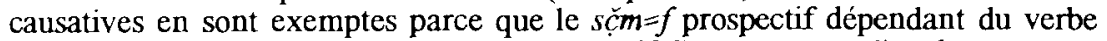
$\check{c}$ i. $t$ est censé ne pas constituer un temps duratif. Par contre, on l'analyse comme unité en démotique tardif, cela explique d'ailleurs que l'on trouve une occurrence de la construction $l$. $i r i=y$ c c $\underline{i} . t-m h n \cdot l m=k$ "je t'allume" (pMag. LL. 6,

${ }^{20}$ La tentative de Elanskaya (1981:108-112) de faire dériver les qualitatifs des verbes causatifs à partir des passifs terminés en tw, me semble difficile à soutenir, pour des détails sémantiques aussi bien que pour des raisons diachroniques: la terminaison passive en tw n'est plus utilisée dès le démotique archaíque, mais les qualitatifs nouveaux des causatifs n'apparaissent qu'en démotique tardif. 
15) avec le médiateur qui aurait été impossible dans un stade plus ancien de langue.

Ce processus de lexicalisation a entraîné une autre conséquence. Si le či.t et le verbe principal sont inséparablement liés, on est confronté à certains problèmes dès lors que l'on a une construction qui exprime le sujet après le verbe $\breve{c} \grave{i}$, c'est-à-dire dans les cas où l'auxiliaire est conjugué avec $s c \breve{m} m=f$. L'ancien modèle serait de former $\check{c} \grave{i}=f$ sçm, mais il devient impossible dans la mesure où $\check{c} \grave{c}$ et $s \grave{c} m=\int$ forment une unité fixe ${ }^{21}$. En copte, on trouve bien sûr des constructions comme afjpof qui correspondrait à $* l r i=f$ chi $t-h p r=f$. Comme toutes les conjugaisons sont périphrastiques, on a de nouveau atteint une stabilité. Entre ces deux extrêmes, le terrain est incertain. La tendance générale est la suivante 22 . Surtout dans les textes du ler et 2ème siècle après J.-C., existe

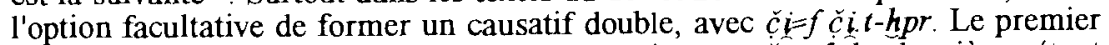
verbe $\check{c} i$ (aussi écrit $t w$ ) est toujours employé avec sçm=f le deuxième étant toujours à l'infinitif ${ }^{3}$. A l'heure actuelle, les sources principales sont les textes littéraires dans les manuscrits romains qui l'utilisent de façon facultative. Ainsi,

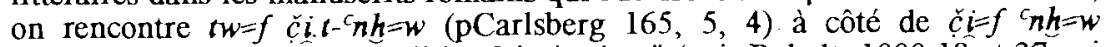
(pCarlsberg 165, 5, 8) pour "il les faisait vivre" (voir Ryholt, 1999:18 et 37 qui émende indûment le texte). La deuxième forme suit le modèle ancien; la première considère $\breve{c}$ l.t. ${ }^{\complement} n h$ comme une unité inséparable, comme tanho en copte.

Cette construction est aussi attestée dans le papyrus magique de Londres et Leyde, voir $h r t w=f$ çi.t hpr "il crée" (pMag. LL. 11, 25; similaire 13, 21) ${ }^{24}$. Mais pour le verbe ćit $i w i$, on utilise déjà une construction périphrastique qui correspond à l'état du copte, par exemple $h r$ lri $i=f c c i . l i w i$ snf $r$-bnr "il verse du sang en dehors" (pMag. LL. vs. 2, 15; sim. 4, 22). De même, on trouve my lr $_{i=f}$ $\check{c} \boldsymbol{i} . t-h^{c} \breve{c r} . \digamma f$ "qu'il étende sa main" en construction périphrastique (pMag. LL. $18,32)$.

La solution à envisager doit tenir compte du processus diachronique, de même que de la divergence entre la date de la composition originale et la date du manuscrit. Je propose le scénario suivant : la construction "double" du

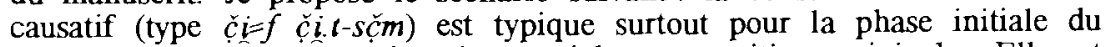
démotique tardif, peu représentée parmi les compositions originales. Elle est

21 Un processus comparable, qui se déroule déjà en démotique ptolémaïque et dans les manuscrits littéraires de l'époque romaine, concerne l'expression $\breve{s} m n=f^{n}$ "s'en aller" qui forme une unité à un tel degré qu'elle ne se laisse plus séparer par un substantif. Par conséquent, on utilise $\breve{s} m=f n=f$ NN en exprimant le sujet d'abord par le suffixe, ensuite en le reprenant par le substantif placé après l'expression complète

22 Voir Depuydt (1998:231-236) mais son exemple allégué de l'époque ptolémaĩque (Setne I 5, 27) est à écarter : il faut plutôt comprendre $t w=s$ tw< $<w>$ lwi $=w$ r hry comme "elle les faisait jeter en bas"; l'orthographe tw montre bien qu'll ne s'agit pas de l'infinitif, qui serait obligatoire dans un vrai causatif double. L'exemple le plus ancien qui m'est connu actuellement est pInsinger 16, 12 .

${ }^{23}$ Pour le verbe $\check{c} i$, les deux formes grammaticales présentent toujours une orthographe distincte

24 Dans le deuxième exemple, $\breve{c} i . t$ est rajouté au-dessus de la ligne. 
produite quelques fois comme rajeunissement quasi-automatique par les scribes de l'époque romaine dans leurs copies des textes littéraires. Sa raison d'être réside dans le fait que la dissociation du causatif $\breve{c} i$. - sčm est censée être inappropriée, mais que la construction périphrastique $i r i=f s c ̧ m$ n'est pas encore généralisée dans l'usage. Dans la mesure où la dernière possibilité devient courante vers l'époque romaine tardive, le causatif double tend à disparaître assez rapidement ${ }^{25}$. En copte, il n'existe plus sauf dans la tendance préférentielle à préfixer $m a$ (impératif du verbe ti) à l'impératif des verbes causatifs (voir Depuydt, 1998:234-236).

\subsection{L'aoriste}

La situation pour l'aoriste est bien plus compliquée que pour le prospectif. Il est nécessaire d'examiner la situation pour chaque verbe séparément.

Verbes toujours utilisés avec la forme simple :

- lwi "venir" (pMag. LL. 3, 24. 27; 5, 31; 3, 25; 19, 32; 28, 15; vs. 19, 7).

- wht "chercher, désirer" (pMag. LL. 13, 16; vs. 15, 1).

- phrr "enchanter" (pMag. LL. 3, 21. 24. 35; 3, 32).

- mwt "mourir" (pMag. LL. 13, 20. 22. 23; vs. 32, 10).

- $m t w$ "parlex" (pMag. LL. 25, 22).

- nw "voir" (pMag. LL. 4, 8. 24; 5, 7. 30; 6, 6; 8, 18; 23, 27; 27, 19. 30; 29, 25; vs. $22,7 f$.$) .$

$-r$ "croitre" (pMag. 14, 31; 24, 22; vs. 4, 16)

- $l k$ "cesser" (pMag. LL. vs. 4,15 ; vs. 11,6 ; vs. 30,10 ).

- $h y$ "tomber" (pMag. LL. vs. 30, 1 )

- hpr "se passet, devenir" (pMag. LL. 9, 16).

- shb "écrire" (pLeyde I 384 vs. II, 13.21 ).

- śm $^{\prime}$ "allet" (pMag. LL. 6, $1 ; 10,9 ; 13,11 ; 18,28 ; 28,4$ ).

- gmi "trouver" (pMag. LL. 5, 25, 5, 27; 27, 25. 27; 5, $28=27,28 ; 21,11$ ).

Verbes toujours employés dans la construction périphrastique :

- wnh "révélet" (pMag. LL. 17, 15; 29, 29).

- $w \breve{c} 3$ "être sain" (pMag. LL. 4, 23).

- mri "aimer" (pMag. LL. vs. 30,4).

- $m k h$ "être en détresse" (pMag. LL. 24, 5).

- lby "être en rage" (pMag. LL. vs. 32, 9).

- $h w y$ "jeter" (oMedinet Madi 10, 12).

_..

- sçy "raconter" (pMag. LL. 3, 22; 4, 20).

- $\breve{s}^{\prime} !$ "couper" (pMag. LL. vs. 3,11 ).

- šni "demander" (pMag. LL. 28, 10).

- $k(r) s$ "ensevellir" (pMag. LL. 13, 29).

"cōnm "être aveugle" (pMag. LL. 13, 12. 26).

- "tur "?" (pMag LL. 13, 25).

- çi.t lwi "envoyer" (4, 22; vs. 2, 15).

${ }^{25}$ Dans le papyrus Krall, on trouve déjà la forme "jeune" lrif=f čit.thpr "il produira" (1, 10) à côté de la forme "intermédiaire" $\breve{c} i=f \breve{c} i . t-s m^{\prime \prime}$ "il étendra" $(1,15)$. 
Verbes utilisant les deux possibilités

- iny "apporter" (simple : 3, 5; 4, 1; 5, 24; 14, 17; 16, 22. 27, 25; 17, 21; 23, 2; 25, $8 ; 25,23 ; 29,1 ; 29,26 ;$ vs. 2,11 . 13; vs. 19,1 ; pLeyde I 384 vs. *II, 7 ; IV, 13 ; III, 2 ; I, 1; périphrastique : vs. 17,5 )

- ir "faire" (simple : 3,$15 ; 10,21 ; 16,28,18,7.21,9.19 .23,31 ; 25,16 ; 25,37$; 27,$19 ;$ vs. 10,12 ; vs. 24,$5 ; 6,6 ; 13,17.14,30 ; 17,16 ; 24,24 ; 24,34.37$. $39 ; 28,8 ; 28,11$; vs. 17,$4 ; 4,1$; pLeyde I 384 vs. $I V, 26$; périphrastique: pMag. LL. 27,$33 ; 29,30$; vs. 3,6 s.; vs. 12 , 4f.; vs. 12,$5 ; 12$, 5s.; pLouvre E $3229,2,27)$

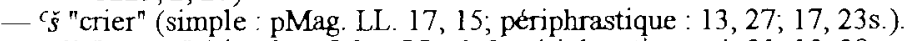

- w3h "mettre" (simple : pMag. LL. 6, 9; périphrastique : 4, 21; 13, 28; vs. 26, 4).

- sçr "dormir, se coucher" (simple : pMag. LL. 23, 6f; périphrastique : 5, 32s.).

- $\breve{c} i$ "donner" (simple : pMag. LL vs. 32,13 , périphrastique : 5,$28 ; 28,11 ; 29,25$ ).

- $\overline{c t} "$ "dire" (simple : pMag. LL : 5,$8 ; 16 ; 14,4 ; 27,17$; vs. 15,5 , périphrastique : $27,36)$.

La plupart des verbes ont donc une formation individuelle fixe, mais il semble possible de trouver au moins quelques tendances pour ceux où les deux possibilités sont attestées.

Le verbe $i r i$ "faire" est toujours un cas spécial, étant donné qu'il est verbe auxiliaire en même temps. Mais une distinction assez nette semble appliquée à l'aoriste: mise à part une exception (pMag. LL. 4, 1), tous les exemples de la forme simple ont un sujet pronominal; de même à une exception près (pMag. LL. vs. $3,6 s$.), tous les exemples de la construction périphrastique ont un substantif comme sujet ou s'appliquent au prédicat composé iri šw "avoir de la valeur", copte $r$-sau $(27,33 ; 29,30)$.

Pour le verbe $w i h$, la seule attestation de la forme simple provient d'un passage archaïque $(6,9)$; tous les exemples qui relèvent vraiment du démotique tardif montrent la conjugaison périphrastique.

Pour $\check{c} i t . t$, la seule attestation de la forme simple est notée en hiératique, elle a donc au moins des chances d'être basée sur un modèle plus ancien.

Pour lny, on a presque toujours la forme simple. La seule exception pourrait provenir d'un passage particulièrement récent.

La fluctuation dans l'usage avec certains verbes montre bien sûr le caractère transitoire du démotique tardif, mais il y a quand même une tendance notable : la forme ancienne est encore usuelle pour certains verbes, et ce sont surtout ceux qui sont fréquents dans la langue parlée. En fait, il est rare de trouver un verbe conjugué de façon périphrastique et ayant plus de deux attestations dans le texte du papyrus magique de Londres et Leyde. Les seuls cas sont $i r i b$, wh th tous fluctuent entre les deux usages - et deux d'entre eux (lri $\vec{i}$ et $\dot{c} i . t)$ ont une prononciation assez réduite dans la forme $s \breve{c} m=f$ qui pourrait inciter à une construction périphrastique. Il n'y a rien de surprenant dans le fait que ce soient les verbes les plus usuels de la langue qui gardent le plus longtemps la forme ancienne.

\subsection{Le passé}

Le passé se rencontre assez rarement dans le texte. On trouve encore la forme ancienne en $s \breve{c} m=f$, mais aussi une morphologie périphrastique correspondant 
au copte afsōtm. Le préfixe est noté $r$ (comme la préposition) devant un substantif et $i w=f$ ou $r$. $w=f$ avec le suffixe de la 3ème personne du singulier; les autres personnes ne sont pas attestées. Les attestations de la forme périphrastique sont largement concentrées dans deux passages, pMag. LL. vs. 20 et vs. 33. On ne trouve ailleurs que des attestations isolées (pMag. LL. 18, $29 ; 20,8(?)$ ) contre bon nombre de passages qui utilisent la forme ancienne simple (pMag. LL. 6, 35 (bis); 9, 32; 13, 2;20, 7; 20, 7; 20, 9; 20, 18; 21, 20). Le corpus ne semble pas suffisant pour une analyse détaillée ${ }^{26}$. Néanmoins, la concentration de la forme nouvelle dans deux passages (qui n'utilisent plus la forme simple) montre bien qu'il faut tenir compte du caractère de compilation et de la non homogénéité du papyrus magique de Londres et Leyde. En dehors de ce manuscrit, on trouve la forme périphrastique dans pLeyde I 394 vs. *I, 2 (seule attestation du passé dans ce papyrus).

Il faut aussi noter le verbe msty "haïr" (et mri "aimer") qui possède la particularité d'utiliser la forme $s \breve{c m}=f$ avec un sens de présent, ainsi que la phrase relative utilisant $n t i$ comme converteur, ce qui est normal pour les formes du présent, mais impossible pour le vrai sčm $=f$ du passé (voir aussi la discussion de Stadler, 2003:111sq). On rencontre encore une attestation de cet usage dans le papyrus magique, à savoir : mt.l lw mst $(=i) s$ "une chose que je ${ }^{27}$ haïs" (pMag. LL. 11, 11).

Il faut noter également la forme du temporalis qui doit être considéré, en démotique, comme conversion du passé, pas encore comme phrase subordonnée selon la situation en copte (voir Quack, 1994a). Dans le papyrus magique, il n'y a qu'un seul exemple, $n$-čr.t gmi=k "après avoir trouvé" (pMag. LL. 6, 31), et ceci provient d'un passage généralement ancien dans l'usage de la langue. On n'a donc aucune attestation qui pourrait nous informer sur des usages spécifiques en démotique tardif.

\subsection{Excursus: La vocalisation de $\mathrm{sç} m=\mathrm{f}$ démotique et la question de son unité}

Dans la discussion ci-dessus, j'ai toujours parlé de $s \breve{c} m=f$ comme entité unique, bien que j'ai distingué plusieurs fonctions. La question est, bien sûr, de savoir s'il n'existait qu'une seule forme morphologique ou bien plusieurs variantes distinguées par la vocalisation ou l'accent. Selon la théorie de Johnson, il serait possible de distinguer trois variantes morphologiques qui correspondent aux trois emplois (passé, aoriste et prospectif; Johnson, 1976:1216). Une vérification de ses exemples laisse cependant un doute. Voici l'analyse dans son détail : la vocalisation de $s c ̧ m=f$ prospectif est bien connue grâce à la fréquence des anciens causatifs lexicalisés en copte. On a toujours la voyelle $o$

\footnotetext{
26 Dans les ostraca de Narmouthis, la construction périphrastique semble être normale (par exemple 7,2), mais là aussi il n'existe pas assez d'attestations pour exprimer un jugement définitif.

27 Selon le sens, il faut certainement comprendre le passage comme ayant un suffixe non écrit de la lère personne du singulier, voir Johnson (1976:72 n. 71).
} 
en sahidique ${ }^{28}$, ce qui indique un ancien $\breve{a}$ accentué dans la syllabe finale. Y a-t-

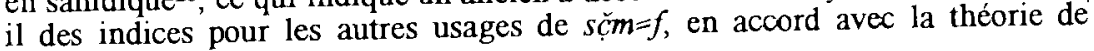
Johnson?

Son premier cas est celui des verbes adjectivaux, qui possèdent une morphologie particulière pour le présent et le passé, formée par le préfixe de $n^{3}$ ou $n \hat{n}$. W devant une forme qui possède l'apparence de sčm=f L'origine étymologique de cette formation est obscure. Son utilisation milite plutôt contre une vraie dérivation de $s c ̧ m=f$, étant donné qu'elle est niée par $b n \ldots$ in, et peut être employé telle quelle dans des conversions (dans la phrase relative après $n t h$, aussi bien que dans le temps second qui est $\{: \ln i n\}-n f r=f)$, ce qui est impossible pour le $s c ̧ m=f$ normal en démotique. Le $s c ̧ c h=f$ des verbes adjectivaux est restreint aux usages du prospectif et de l'aoriste. Mais ceci est une restriction de la fonction, qui ne tranche pas la question de savoir s'il existe une différence morphologique entre le passé et le prospectif ou non

Le deuxième point de Johnson est un élément $t$ qui apparaît, selon son argumentation, après trois verbes dans les instructions d'Ankhchechonqi. II n'apparaît pas clairement pour moi dans quelle mesure cela peut servir à établir une distinction entres les usages de $s \check{c} m=f$. En fait, de ses trois cas, l'un est erroné $^{29}$, et les deux autres ne concernent que des verbes à dentale comme dernière consonne forte où le $\mathfrak{l}$ sert à signaler la préservation de la dentale dans la prononciation ${ }^{30}$

Ensuite Johnson invoque la morphologie spéciale du verbe $r h$ "savoir", qui a toujours $r h$ au passé, mais pour lequel, au prospectif, est aussi orthographié ir.rh, et cette orthographe est normale pour l'aoriste négatif, dont on connaît la vocalisation par le copte mešak "peut-être" (étymologiquement: "tu ne sais pas"). Plusieurs problèmes se posent. D'abord, l'orthographe avec un ir supplémentaire concerne un problème de consonnes: la racine tend à perdre irrégulièrement le $r$ initial, et l'élément $i r$ sert à le renforcer graphiquement là où il est encore prononcé. Mais cela n'a rien à voir avec la vocalisation, et en tout cas, la graphie ir.rh pour le prospectif est bien trop rare pour pouvoir être considérée comme régulière. L'aoriste négatif $b w r h=k$ est une formation particulière de ce verbe, les autres verbes démotiques utilisant la forme périphrastique $b w$ ir $i=f s c ̧ m$. La forme copte mešak indique un ancien $\breve{\imath}$ ou $\breve{\iota}$ dans la syllabe finale. On a donc bien une vocalisation particulière pour cette formation, qui est différente de celle de $s \grave{c} m=f$ prospectif, mais elle n'a rien à voir avec la vocalisation des usages de $s \breve{c m}=f$ régulier des verbes démotiques.

28 Osing (1976:175sq. n. 268) a essayé de démontrer l'existence d'une vocalisation en $\breve{e}$ pour le prospectif au moyen du verbe $j(e)$ na "étemdre" mais ceci est plutôt à expliquer comme un dérivé de çît. $\breve{s} m n=$ en suivant Černý (1971:44-46); la voyelle est donc celle de la préposition, et non pas celle du verbe.

${ }^{29}$ Dans Ankhchechonqi 15, 19, il faut comprendre bn lw shn $\models k$, avec $t k k$ comme pronom enclitique de la 2 ème personne du singulier.

30 Pour sçr dont la prononciation à cette époque était plutôt quelque chose comme šte (voir Quack, 1996, cl. 154, remarque pour V, 25), on trouve aussi le / pour l'infinitif dans Ankhchechonqi 2, 18. 
La distinction suivante est proposée pour le verbe wn "être", pour laquelle Johnson distingue entre une forme indicative $w n$ et une forme wn-niw à l'imparfait. Néanmoins, cette distinction ne concerne absolument pas $s \breve{c ̧ m} m=f$. L'élément wn ne peut pas être considéré, dans une analyse synchronique, comme une forme $s \grave{c} m=f$. Selon son usage en démotique, il n'est que l'expression de l'existence (servant aussi comme introduction des substantifs indéfinis dans les temps duratifs). L'élément $w n-n 3 . w$ aussi n'est pas une forme $s c ̌ m=f$ dans une analyse synchronique, mais un convertisseur de l'imparfait. La distinction des ces formes, toutes deux devenues des morphèmes grammaticaux, n'a aucun rapport avec la vocalisation des usages de $s c \breve{m}=f$.

Finalement, on arrive à des cas où une vraie distinction vocalique est proposée ${ }^{31}$. Mais pour l'apprécier, on doit tenir compte du problème de la valeur du graphème démotique qui est aujourd'hui transcrit $e$. Il est formé par deux petits traits verticaux. Dans bien des cas cependant, il ne correspond pas à une intention de marquer une voyelle, mais tire son origine de ligatures différentes (Sethe, 1927). C'est donc un indice peu sûr, et il faut toujours vérifier s'il est présent dans toutes les graphies du verbe ou seulement dans des formes nettement distinctes.

Johnson énumère un certain nombre de verbes qui ont ce $e$ dans la forme prospective devant un sujet nominal. Mais hélas, cela n'a pas de valeur pour établir une distinction dans la prononciation. En copte, les causatif lexicalisés, qui possèdent o comme voyelle accentuée, le changent en $\epsilon$ devant un substantif parce que l'accent principal de cette unité porte sur le substantif. L'orthographe démotique confirme ce qu'on aurait supposé d'ailleurs, à savoir qu'une voyelle non accentuée se réduit dans la prononciation, mais elle ne nous aide pas à différencier les formes de $s \check{c} m=f$.

Reste l'argument final, la vocalisation en $e$ pour l'aoriste avec un suffixe comme sujet. Il se base sur deux indications distinctes. L'une est la présence du $e$ démotique. Johnson indique quatre verbes pour lesquels on trouve ce $e$ dans l'aoriste avec un sujet pronominal. L'un d'entre eux peut être refusé immédiatement, à savoir $r$ "croîre". Pour ce verbe, le e supposé n'apparaît pas seulement à l'aoriste $(14,31,24,22$, vs. 4, 16), mais aussi à l'infinitif (vs. 33, 6), donc dans toutes les formes attestées de ce verbe. Et pour l'infinitif (qui est rōt en copte), il peut être exclu qu'il y ait une voyelle $e$ terminale. Le deuxième cas, à savoir whe "désirer", n'est pas mieux établi. Normalement, ce verbe apparaît dans le papyrus magique avec une graphie relativement abrégée qui n'indique en rien la présence d'une voyelle $e$. Il existe seulement quatre cas avec une graphie plus longue, et dans ces quatre cas, le signe supposé est $e$. Un cas (vs. 15,1) est un aoriste, les trois autres (vs. 12, 4 et 5 (bis)) un infinitif. Cette fois, l'usage dans l'infinitif pourrait se justifier par la forme Awōxe. Mais on manque de graphies opposées qui pourraient prouver que la présence du $e$ est typique pour certaines formes à l'exclusion des autres. Le troisième verbe à considérer est $p h r$. Là, on a deux attestations de l'aoriste sans marque vocalique

31 Pour la question de vocalisation des formes $s \breve{c} m=f$, voir dernièrement Osing (1998:6264). 
(hr phr $=f$ pMag. LL : 3, 24. 35), alors que deux autres fois, on a $h r$ phre $=f$. (pMag. LL. 3, 21. 32). Le seul cas du verbe $p h r$ au $s c ̧ m=f$ prospectif est noté $m y$ $p h r=f$ sans $e$ (pMag. LL. 18, 9). Finalement, on trouve $h r h p r e=f$ (pMag. LL. 9, 16), le seul cas où ce verbe est à l'aoriste. Les graphies démotiques pour $e$ à l'aoriste se réduisent donc à trois attestations (de deux verbes), ce qui est bien pell.

L'autre indice est l'écriture cryptographique de certains mots. Ce système cryptographique semble avoir été conçu pour cacher les indices les plus sûrs du caractère illicite des recettes ou pour garder secrètes les drogues nécessaires pour obtenir les effets désirés. Parmi les mots notés avec ce système, se trouve la conclusion suivante "alors il meurt" ou "alors elle meurt". Selon l'interprétation aujourd'hui admise, les cryptogrammes en question sont à lire mtef et mtes (voir Griffith \& Thompson, 1904-09, vol. III, p. 110 , numéros 45-47). Ils donneraient alors une vocalisation en $e$ pour la syllabe accentuée. Malheureusement, la précision de la valeur vocalique me semble assez peu assurée. Seuls deux signes cryptographiques sont concernés ${ }^{32}$. Sur quels indices ces valeurs sont-elles assignées, et quelle précision atteignent-ils ?

Le premier signe est défini comme $e$ d'une part à cause du fait qu'il se trouve très fréquemment à la finale d'un mot, ce qui correspondrait à la terminaison en $\boldsymbol{e}$ de beaucoup de mots coptes. L'autre argument est qu'il y a un échange avec l'autre signe en question ici. Celui-là, à son tour, est justifié par son usage dans le mot *smune "canard" et par l'échange avec un autre signe défini par un mot qui est attesté avec un $e$ en écriture démotique normale.

Je ne pense pas que ce déchiffrement soit tout à fait erroné. Dans nombre de cas, les chiffres correspondent en fait à un $e$ (soit long, soit bref). Néanmoins, il semble qu'il y ait une bonne marge de latitude dans l'emploi de ces signes vocaliques. La liste suivante servira à montrer des cas où une lecture différente s'impose.

Voyelles accentuées ${ }^{33}$ :

*en (no. 8) et *een (no. 14); les formes sahidiques $(\bar{e} n(e)$ ) et bohairiques $(e n)$ pourraient correspondre, mais la forme vieux-copte qui devrait être la plus proche de la langue du papyrus magique ${ }^{34}$ est ana.

* $m$ seh (no. 43 et 44 ); en copte, on a ${ }^{s} m s a h$, et le pluriel msooh montre bien que la voyelle dérive d'un ancien $a$.

* ches (no. 90 et 91); en copte, on a "chos.

Par contre, il existe 14 cas où un $e$ (bref ou long) en syllabe accentuée est certain et régulier (no. $5,6,18,26,28,38,50,59,68,71,75-77,79$ ).

\footnotetext{
32 Les numéros iii et iv de la liste Griffith \& Thompson, 1904-09, volume III, p. 105, où l'on trouve aussi les arguments pour la lecture.

${ }_{33}$ Les numéros entre parenthèse indiquent la numérotation dans la liste de Griffith \& Thompson 1904-09, volume III, p. 109-112. Jai accepté comme réguliers les cas ou un $e$ accentué de l'akhmimique, fayoumique ou lykopolitain pourrait correspondre à un d sahidique ou bohairique.

34 En fait, la forme est attestée dans PGM IV 105, un manuscrit qui a été trouvé avec le papyrus magique de Londres et Leyde.
} 
Voyelles non accentuées :

* beempe (no. 2); en copte on a baampe ou sim.; aucun dialecte copte ne possède be en début de mot.

* bešs (no. 4); en copte, on trouve baššs.

*ebōk (no. 7); en copte, c'est $a b \bar{k} k$ que l'on rencontre; tous les dialectes possèdent un $a$ initial.

* emis (no. 12); le sahidique, qui a emise, correspondrait à cette forme, mais en bohairique, on trouve amise.

* erekos (no. 17); ce mot semble correspondre au grec ápaxos.

* cele (no. 83); en grec raìn.

* cemul (no. 85); en copte, on trouve camul, avec a dans tous les dialectes.

L'évaluation de la situation pour les syllabes non accentuées dépend fortement de suppositions pour les voyelles finales. Si elles sont en $e$, on aurait une bonne majorité pour l'usage des signes tels que $e$.

De plus, on est également confronté au problème du mot signifiant "bile" qui est attesté comme ${ }^{*}$ saxe aussi bien que comme ${ }^{*}$ sexe, donc avec une variation entre le signe dont on est peu sûr et le signe indubitable $a$. Ce même échange se retrouve dans *haflela (no. 75) à côté de *heflele (no. 77).

Tout cela montre au moins que la valeur $e$ pour les signes en question n'est pas établie avec assez de certitude pour pouvoir exclure qu'ils servent à indiquer un $a$ étymologique. Il existe trop de cas où ils indiquent ce son, ou bien sont en combinaison complémentaire avec un signe notant $a$. Mais justement s'ils peuvent aussi indiquer le $a$, c'est précisément la vocalisation connue pour $s c ̧ m=f$ prospectif. Donc, tant que l'on ne peut pas exclure que les signes cryptographiques en question peuvent aussi avoir la valeur $a$, les graphies concernées ne peuvent pas être utilisées pour prouver que la vocalisation de l'aoriste était différente de celle de $s \check{c} m=f$ prospectif.

De plus, certains indices montrent qu'une vocalisation en $e$ n'était pas tout à fait inconnue dans la forme prospective, si les noms propres $\varepsilon \tau \varphi \varepsilon \nu \varsigma$ (ég. ht $p=w$ ) et equeus (ég. $h r i-w$ ) sont bien à considérer comme des prospectifs en fonction optative, et non comme des qualitatifs ${ }^{35}$.

Finalement, une distinction entre aoriste et prospectif semble un peu difficile à établir pour une raison étymologique. Il y a de fortes chances que l'usage de $s c ̧ m=f$ à l'aoriste soit dérivé de l'emploi du prospectif (Winand 1992:232-234).

Par contre, sous l'aspect de l'économie de la langue, il n'y a pas de nécessité de distinguer les fonctions de $s \check{c ̧}=f$ démotique par la vocalisation, parce qu'elles sont suffisamment marquées par leur position. L'aoriste est facile à reconnaître à cause du préfixe $h r$, et le prospectif est restreint, en démotique normal, à l'usage après le verbe $\breve{c} i$ "causer" ou bien pour continuer une forme injonctive. Dans tous les autres cas, le passé s'impose. On ne peut pas exclure

${ }^{35}$ Ainsi Osing (1976:174 n. 266sq); Osing (1998:64). Les autres exemples allégués par Osing (1976:174-178) ne sont pas valables, en particulier hna qui n'est pas du tout un ancien $s c ̧ m=f$, voir Zauzich (1977:152); ceci est reconnu aussi par Osing (1998:65 n. 278). 
que des distinctions plus anciennes perdurent en démotique, mais elles ne sont plus nécessaires, et une neutralisation de la prononciation est possible.

\section{LES PHRASES RELATTVES DU PASSE}

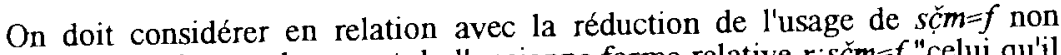
périphrastique le remplacement de l'ancienne forme relative $r: s c ̧ m=f$ "celui qu'il a écouté" et du participe $l:\{r i$ sčm "celui qui a écouté"36, par une forme nouvelle de la phrase relative du passé, à savoir ntafsōtm en copte. En fait, les deux formes ne fonctionnent pas tout à fait ensemble. Alors que la forme relative a complètement disparu en copte (sauf un vestige lexicalisé : voir plus bas) ${ }^{37}$, le participe a encore un emploi considérable sous la forme relictuelle ersōtm (voir Shisha-Halevy, 1983:315sq., et Schenke, 1991:53 avec n. 111).

L'orthographe normale pour la nouvelle formation de la phrase relative du passé en démotique tardif est $n t l r$ (pour ${ }^{*} n t a$ ), par exemple ntl $r i: i r=k$ ir $i=f$ "ce que tu as fait" (pMag. LL. 5, 14; autres exemples en 12, 26; 21, 26; 21, 27; vs. $12,6)$. Avec un suffixe, on a $n l l, r l: l r=k m h r^{\prime}=k r . r=f$ "celui que tu as mordu" (pMag. LL. 19, 37), ce qui semble être une graphie tout à fait normale pour *ntak.

On rencontre tout de même, dans une seule formule du texte, plusieurs exemples d'une construction étrange, qui semble être antérieure à ce stade de langue et qui est caractérisée par la combinaison du $n t l r$ avec une forme de

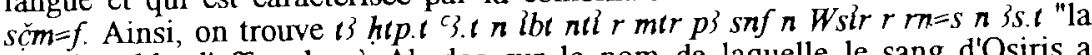
grande table d'offrandes à Abydos sur le nom de laquelle le sang d'Osiris a témoigné pour Isis" (pMag. LL. 15, 13); p3 mr nti $r$ lri s 3s.t $r$ Wsir "l'amour qu'Isis avait pour Osiris" (pMag. LL.15, 16); $p^{3} p \breve{c}$ nil $r$ lri s hw.t-(hr)(?) $r$ Hr $B h t$ "la prédilection qu'Hathor(?) avait pour Horus d'Edfou" (pMag. LL. 5, 18). Bien qu'inattestée ailleurs, cette forme me semble logique. Le copte ntafsōtm est visiblement une transposition de la forme de base afsōtm au moyen du converteur $n t$; elle suppose donc étymologiquement un $n t i l$ ir $i$ f sčm; et de fait, nous avons déjà vu que le signe $r$ devant un substantif était une forme normale du passé. Ici, la forme $n i l r s \breve{c} m=f$ semble constituer un stade intermédiaire.

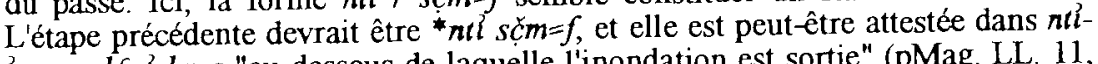
lw pyr $h^{c} p l h r=s$ "au-dessous de laquelle l'inondation est sortie" (pMag. LL. 11, 5f.) mais la lecture et l'interprétation de ce passage sont peu sûres.

La phrase relative du parfait est différente, mais en relation fonctionnelle avec celle du passé. On a une attestation écrite $n t i-l w w 3 h=k$ chit $h p r p 3 k k e p^{3}$ wyn n\}.e-hr=f "celui devant lequel tu as crée l'obscurité et la lumière" (pMag. LL. 5, 14), qui correspondrait à * ete hakjpe pkake pwoin nahraf. Dans ce cas, il $n^{2} y$ a pas de changement profond entre le démotique et le copte.

\footnotetext{
${ }_{36}$ Malgré l'opinion contraire de Depauw (2002), elle est toujours au passé, et non pas au présent; une argumentation détaillée sera donnée ailleurs.

37 Il en existe encore en vieux copte (qui est sans aucun doute du démotique tardif écrit en lettres coptes).
} 
Il faut noter aussi une seule attestation, où l'antécédent est en même temps le sujet de la phrase relative, c'est-à-dire où on aurait utilisé un participe en démotique classique : šy. $\neq k n t l r r h$ wnm(?) p s sym "ton nez qui savait manger les herbes" (pMag. LL. 21, 21). C'est un peu étrange; en copte on aurait soit la forme conjuguée avec ntaf, soit une forme fixe ntox, etah ou une forme similaire (typique pour le dialecte lycopolitain). Peut-être faut-il remarquer qu'il y a aussi des variantes utilisant nta $\not$, eta ${ }^{\zeta}$ ou tas dans des textes coptes non littéraires (Kahle, 1954:177-179). La graphie $n t i l r$ rh pourrait peut-être représenter * $n t a s ̌$, même si la distance chronologique est considérable. Mais on ne peut pas exclure qu'il s'agisse simplement d'une tentative pour mettre la phrase relative du présent I (avec pseudoparticipe) au passé.

L'ancienne forme relative apparaît aussi dans le papyrus magique de Londres et Leyde. Trois des exemples se trouvent dans une section qui est certainement une des plus anciennes du manuscrit $(6,13($ bis $) ; 6,35)$; trois autres dans des sections également traditionnelles, probablement pas vraiment du démotique tardif $(10,7 ; 11,4 ; 11,16)$. Du reste $(20,7 ; 20,23 \mathrm{f} ; 27,16)$, on peut discuter l'ancienneté des passages en question, mais ils ont au moins des chances d'être dérivés de modèles plus anciens.

On peut résumer les résultats ainsi : l'échange de la forme relative démotique par la conversion du passé est achevé en copte et déjà bien avancé en démotique tardif; lorsqu'on utilise encore la forme relative, c'est normalement sous l'influence des archétypes anciens. Par contre, le remplacement de l'ancien participe, qui est fort avancé en copte, mais avec des vestiges considérables du stade ancien, a à peine commencé en démotique tardif, qui apparaît une fois de plus comme un stade transitoire.

On rencontre encore un vestige lexicalisé de la forme relative en copte, à savoir la forme pejaf "il a dit", qui dérive de la formation démotique $p\} r$ : $̌ c t=f$. Son usage comme forme verbale normale pour introduire un discours direct est asse $z$ tardif et non régulière en démotique. Il y en a un exemple dans le démotique tardif du papynus magique : p3e-čte kli čm $\mathrm{cm}^{c}$ "un autre livre a dit" (pMag. LL. 18, 10). En fait, les attestations de ce cas sont déjà nettement plus anciennes, par exemple dans le papyrus Krall (écrit sous Hadrien) $p \nmid l-c ̧ t=w$ "on a dit" (pKrall 2, 17) . $^{38}$.

\section{LA DISPARTTION DE L'INFINITIF DANS LA PHRASE COUPEE}

En démotique, il y a une construction bien attestée, qui utilise l'infinitif nu avec une phrase relative suivant la construction de la phrase coupée. Elle peut être mise au passé, au présent ou au futur selon la forme de la phrase relative utilisée (voir Quack, 1991 avec des ajouts dans Quack, 1994b:64 n. 7; Quack, 2000b:170f; Depuydt, 1994:49-73). Cette construction n'est plus attestée en copte. En fait, elle semble être déjà sortie de l'usage en démotique tardif. Les seules attestations dans le corpus concernent des passages qui, pour plusieurs

38 Voir Hoffmann (1996b:366 n. 2230). Un autre exemple est probablement oMedinet Madi 13, 2s; 4s. 
raisons, doivent être considérés comme assez anciens (pMag. LL. 6, 14f.; 6, 37; pLouvre E 3229 1, 23(?); 5, 7). On trouve encore une construction assez semblable dans $\breve{s l l} p\}^{3} n t-i w=y$ ir $i$ n. $i m=f$ "je prie" 10,24$)$, mais l'utilisation du médiateur devant le complément d'objet direct dans la phrase relative ne correspond pas à l'usage spécifique de l'infinitif dans la phrase coupée où il est utilisé avec l'infinitif (dans des constructions transitives), mais pas dans la phrase relative (qui est grammaticalisée).

\section{LES CONSTRUCTIONS NON-VERBALES}

\subsection{La position de la préposition $\mathrm{n}$ avec un suffixe}

Il existe une règle très ancienne de la grammaire égyptienne, selon laquelle la préposition $n$ "pour" suivie d'un suffixe est enclitique et doit être placée avant un substantif sujet, et avant un complément d'objet direct. Cette règle n'est plus vivante en copte, et elle commence à ne plus être appliquée en démotique tardif ${ }^{3}$. Dans le papyrus magique de Londres et Leyde, l'ordre traditionnel des mots est attesté dans environ 62 cas. Pour l'usage non enclitique, il y a environ 20 exemples, les trois quarts des cas pour l'usage ancien. On doit noter aussi que tous les exemples de l'expression č̣t $w 3 h$ "répondre, donner un renseignement" (qui apparaît très fréquemment dans le texte) suivent l'ordre des mots traditionnel.

\section{2. Le médiateur du complément d'objet direct}

Pour le copte, on sait depuis longtemps que le complément d'objet direct peut être rattaché au verbe soit directement, soit au moyen du médiateur $n / m m o=$ (selon la fameuse règle de Stern-Yernstedt). Le médiateur est obligatoire pour des objets syntaxiquement définis avec les temps duratifs, mais ailleurs il est optionnel.

En démotique, la règle est généralement plus stricte. Le médiateur est obligatoire pour les temps duratifs mais il n'existe pas dans les autres constructions ${ }^{40}$. En démotique tardif, la règle se relâche et le médiateur apparaît occasionnellement même avec les temps non duratifs. Ainsi, on a l'optatif $m y$ iri

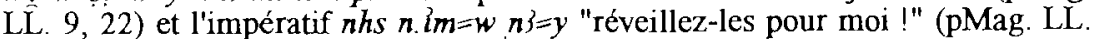
$10,7)$; hbke $\left.\left.n . l m=s{ }^{3}\right\}_{i} n t\right\} y=s$ kt.t "affaiblissez-la, prenez son sommeil" (pMag. LL. vs. 13, 2). Cet usage reste encore très rare, alors qu'il est très fréquent en copte.

39 Une discussion très détaillée du processus est donnée par Depuydt (1997) avec des ajouts dans Depuydt (1998b:64), Depuydt (2002:112sq.).

40 Pour la discussion, voir Simpson, Demotic Grammar, p. 151-156, avec toutes les références à la discussion antérieure, et additionellement Quack (1997/98:175sq.). 


\subsection{La morphologie du pronom enclitique}

En démotique, des pronoms enclitiques sont utilisés comme complément d'objet direct après des formes conjuguées du verbe (impératif, $s \check{c} m=f$, forme relative). Pour la première et deuxième personne, ils sont formés sur une base $t$ suivie du suffixe, pour la troisième personne, on a $s$ au singulier et $s t$ (écrit aussi $s . w$ dans le papyrus magique) au pluriel. Ils ont presque disparu en copte, parce qu'il n'existe plus de formes verbales conjuguées.

En démotique tardif, la situation est passablement compliquée. D'un côté, la réduction de l'usage des formes verbales conjuguées rend les pronoms enclitiques moins fréquents bien qu'ils restent nécessaires pour le fonctionnement de la langue. D'un autre côté, la morphologie tend à s'assimiler à celles des pronoms suffixaux, ainsi de la construction $h r g m i=k f$ "tu le trouves" (pMag. LL. 5, 28; sim. 3, 15;13,17;6, 7; 14, 4; 14, 30;16, 28; 18, 7; $19,20 f ; 21,9 ; 21,19 ; 23,31 ; 24,33 ; 25,16 ; 25,37 ; 27,19 ; 28,11$; vs. 2,11 . 13 ; vs. 10 , 12 ; vs. 24 , 5; vs. 15, 5; pLeyde I 384 vs. IV, 26; voir Depuydt, 2002:108). En fait, pour l'aoriste la documentation est unanime à montrer qu'on utilise $f$ (et non pas $s$ ) comme pronom enclitique de troisième personne masculin singulier; les autres personnes ne sont pas attestées.

On trouve aussi des attestations sûres de l'emploi de $f$ après l'impératif (où l'usage traditionnel demanderait l'emploi du pronom enclitique), dans $r$ :iny $w^{c}$ irp $r-h n k l p=f r n\} n \boldsymbol{X} r . w$ "apporte du vin et ouvre-le pour les dieux" (pMag. LL.

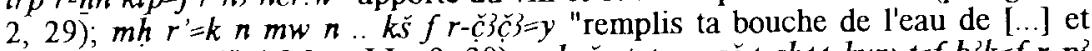
verse-le sur moi" (pMag. LL. 9, 30); $m h$ ç r.t $=$ in $n$ sç.t sht.t hwy. $f f b 3 k=f r p\}$ h3. $n m n$ r:msi $m n$ "remplissez vos mains du feu ardent, jetez-le, utilisez-le contre NN que NN a enfanté !" (pMag. LL. vs. 13, 1). A la lumière de ces exemples, on peut aussi considérer la construction wnh $k$ "révèle-toi" (pMag. LL. 2,$20 ; 5,15 ; 7,10 ; 14,7$ ) comme un impératif, et non comme une forme $s c \breve{c}=f$ prospectif. Cet emploi se rencontre déjà dans les ostraca de Narmouthis, où on a $\check{s} \boldsymbol{n} y$. $f$ "demande-le !" (oMedinet Madi 37, 1. 15; voir Quack, 1999:194) et probablement $m y=f$ "donne-le !" (oMedinet Madi 6, 8). Il n'est pas forcément à interpréter comme utilisation de $f$ comme pronom enclitique. On pourrait aussi le justifier par le fait que l'impératif a été formellement identifié à l'infinitif (Edgerton 1932:64).

Il existe néanmoins une attestation de l'impératif qui garde l'usage ancien: twn $t k$ "lève-toi" (pMag. LL. 20,4).

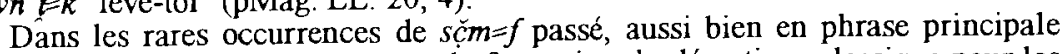
qu'en phrase relative, on conserve la formation du démotique classique pour les pronoms enclitiques, ainsi $r$. In $i=y \neq k$ "je t'ai apporté" (pMag. LL. 21, 20) ${ }^{41}$ et $p$ 3 $m r n i$ r iris 3 s.t "l'amour qu'Isis a fait" (pMag. LL. 15, 17).

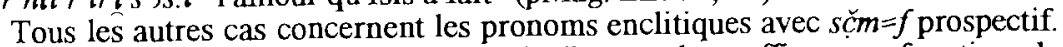
Ici, on n'a pas d'attestation certaine de l'usage des suffixes en fonction de pronom enclitique. En fait, deux phrases sont problématiques et doivent être

4i Le $r$ à l'initiale n'est probablement pas morphologique, mais phonologique, pour montrer que la première syllabe contient un $a$, à la différence de l'infinitif où on a $i$. 
analysées dans le détail ${ }^{42}$. On trouve $m t w=k$ či.t $s w r=f p 3 r m \bar{c}$ "et tu fais que l'homme le boit" $(19,20 \mathrm{~s}$.) et $m t w=k$ čit $w n m=\tilde{f} s p 3 r m \breve{c}$ "et tu fais que l'homme le mange" (pMag. LL. 13, 12). Depuydt propose de regarder le $f$, dans ces cas, comme un complément d'objet direct, c'est-à-dire comme remplacement de l'ancien pronom enclitique, et cette interprétation entraîne que le $s$ en 13,12 est "superflu" (Depuydt, 2002:104s. n. 5). On peut en douter. Dans la langue plus ancienne ( $\mathrm{y}$ inclus le démotique classique), $m t w=k$ či.t $w n m=f$ aurait nécessairement été compris comme "et tu fais qu'il mange", avec $f$ comme expression du sujet mangeant, ainsi aussi en copte, nktmmof "et tu l'alimentes" aurait signalé la personne alimentée par le $f$. Il est extrêmement improbable qu'entre ces deux stades, on aurait admis une construction où le $f$ désignerait au contraire la chose mangée. Il faut chercher une autre solution. À mon sens, $m t w=k$ či.t wnm=f $s$ p $3 r m d$ (pMag. 13, 12) est à interpréter à la lumière de $m t w=k$ çi.t $s w r=f s$ "et tu le fais boire" (pMag. LL. 19, 40), où la personne qui mange n'est exprimée que par le suffixe. $p^{3} r m \tilde{c}$ est donc simplement un substantif en apposition à un suffixe ${ }^{43}$. Quant à $m t w=k$ çi.t $s w r=f p 3 r m \not c$ (19, $20 \mathrm{~s}$.), il est probablement à comprendre comme $m t w=k$ či.t $s w r=f\langle s\rangle p\} r m \bar{c}$, le pronom enclitique ayant été oublié en passant d'une ligne à l'autre; donc un cas identique au précédent.

Dans toutes les autres attestations, par exemple $r \check{c}$ i.t $m\} \xi=f s$ "pour le lui faire voir" (pMag. LL. 7, 16), il n'y a pas de difficulté à comprendre les graphies comme étant celles des pronoms enclitiques normaux du démotique.

Somme toute, on peut analyser la forme qui suit l'impératif comme un exemple du suffixe dû à la restructuration de la syntaxe, qui assimile entièrement les impératifs aux infinitifs. Mais la disparité de l'aoriste, de $s c ̧ m=f$ au passé et au prospectif reste difficile à expliquer. Peut-être est-elle due à la nature un peu fossilisée et figée des constructions causatives qui utilisent encore directement $s \check{c} m=f$ et pourraient donc avoir conservé l'ancien système plus longtemps.

42 Voir la discussion de Depuydt (2002:103-107). Ses exemples "Mode 2" et "Mode 5" n'entrent pas dans les développements de la langue étudiés ici, mais je doute néanmoins de son analyse. À mon avis, dans le cas en question, on peut osciller entre l'expression par le complément d'objet direct $m t w=k$ čit swr $s p$ \} $r m c^{~ " e t ~ t u ~ f a i s ~ q u e ~ l ' h o m m e ~ l e ~ b o i t " ~}$ (pMag. LL. 13, 22, sim. 15, 23; 20, 33; 21, 17; vs. 32, 9) et l'expression partitive $m t w=k$ çi.t swr p\} $r m \dot{c}$ n.n.lm=f "et tu fais que lhomme en boit" (pMag. LL. 13,11s.), avec $m i w=k$ çi.t swr s $t\}$ shm.t n.lm $=f^{n}$ et tu fais que la femme en/le boit" (pMag. LL. 15, 7) comme conflation des deux options. L'idée de Depuydt, à savoir que le n.lm=f est un complément d'objet direct avec médiateur, me paraît peu probable; le médiateur est très peu attesté dans le papyrus magique en dehors des temps duratifs, et le seul exemple d'un causatif lexicalisé avec médiateur apparaît clairement dans un temps duratif (voir plus haut)

${ }^{43}$ Depuydt doute de l'existence de cette construction pour le démotique, mais voir les exemples chez Hoffmann (1995:56sq. n. 163), bien que son point de départ (Amazones $3,28)$, aussi bien que Spiegelberg $(16,12 ; 17,7)$ et Mythus Leyde $(10,29$ sq.) soient à expliquer simplement selon ma note 21 plus haut. 


\subsection{L'ordre des mots dans la phrase nominale}

En démotique traditionnel, l'ordre normal des mots dans la phrase nominale est celui qui place la copule à la fin de la phrase; donc la formation $A$ p 3 pour "c'est A"44 et $A B$ p 3 pour "A est B" (Johnson, 1981:414-430). La juxtaposition de deux noms (type $A B$ ) est assez rare. La position médiane de la copule, donc $A$ p $3 \hat{i} B$, est très exceptionnelle (elle existe quant même, voir pSpiegelberg 11 , 15; plnsinger 30,19), sauf dans un cas particulier : si l'élément B est un pronom démonstratif, l'ordre qui semble être obligatoire est $A p j l p i y$ "ceci est $A^{\prime \prime 45}$. En copte, par contre, l'ordre $A$ pe $B$ est fréquent.

En démotique tardif, la première impression est que la construction récente est la plus fréquente. En fait, les choses sont passablement plus compliquées. Il y a un cas où l'élément $\mathrm{B}$ est un pronom démonstratif : $p 3 y=f^{c_{\breve{s}} n m t, t-n-k m y}{ }^{c_{n}}$ p $3 l$ p $3 y$ "ceci est encore son invocation en égyptien" (pMag. LL. 15, 28); cela n'est pas diagnostique. Pour l'expression fréquente "[...] est mon nom", il existe deux possibilités distinctes : l'une est d'utiliser le mot $r n$ "nom" avec un suffixe; dans ce cas, l'ordre A B sans copule semble être obligatoire, par exemple sčm $m . y=t$ "Écouter est mon nom" (pMag. LL. 2, 13; vs. 27, 1). Par contre, si l'on utilise le pronom possessif dérivé de l'article suivi de l'ajout $n$ mte "vrai", l'ordre A $p\}$ B est préféré (attesté 2,$12 ;$ vs. 27,$2 ; 2,13 ; 9,14 ; 18,12 ; 18,13 ; 19,27$; 20,29 ; vs. 27,$7 ; 23,26$; sim. probablement aussi 23,28 ), bien que l'ordre A B reste possible $(2,13 ; 23,29)$.

En dehors de ces cas spécifiques, on trouve trois attestations de l'ordre A B

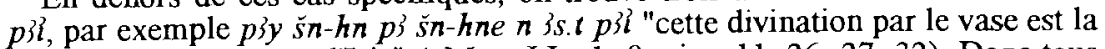
divination par le vase d'Isis" (pMag. LL. 1, 9; sim. 11, 26; 27, 32). Dans tous les cas, le prédicat semble être l'élément $B^{46}$. L'autre ordre se trouve dans $p 3 y=f$ pjl piy $k h$ "le sien est cette terre" $(21,7)^{47}$. Cette fois, le prédicat est probablement l'élément $A$. L'ordre traditionnel du démotique est donc encore un peu plus fréquent si on fait exception d'un idiome spécifique pour indiquer le vrai nom des entités magiques. De plus, il pourrait être possible d'établir une distinction plus nette. On utilise l'ordre A B p 33 si on veut mettre le sujet au début, et $\mathrm{A}$ p $3 l \mathrm{~B}$, si on l'on veut commencer par le prédicat. Les attestations ne contredisent pas une telle idée, mais le corpus semble être trop restreint pour un jugement définitif.

Ceci concerne les cas de la troisième personne, mais il y a aussi des phrases nominales à la première et à la deuxième personne. La solution normale en démotique est d'utiliser les pronoms indépendants sans autre copule. Dans le papyrus magique, c'est aussi la construction largement prédominante.

44 Cette construction se rencontre bien sur dans le corpus, mais comme elle n'est pas significative pour le développement diachronique, elle n'est pas étudiée ici en détail.

45 L'orthographe démotique permet de distinguer le pronom démonstratif de la copule, au moins à partir de l'époque ptolémaique.

${ }_{46}$ En prenant le terme "prédicat" au sens de Polotsky (1987:17-19); son sigle Z.

47 La phrase $m w l^{-c}$ - $t$ p $3 l$ p $l_{r y} g^{c} m(18,13)$ est difficile, peut-être qu'il faut comprendre "c'est Moulôt, celui qui a de la force". 
Néanmoins, on a aussi la possibilité de construire $l n k p \nmid l \mathrm{~A}$ "je suis $\mathrm{A}^{\prime \prime}$ ou $m t w=k$ p jl A "tu es $\mathrm{A}^{n}$, donc de suivre le modèle de la phrase nominale avec copule. La formation $i n k$ A $p j l$ avec position finale de la copule n'est attestée qu'une fois dans le corpus : $i n k{ }^{2} r^{-}{ }^{-} n w$ p $p l$ "je suis celui au beau visage" (pMag. LL. 18, 10).

Une distinction claire dans l'usage semble difficile à donner, toutes les attestations apparaissent dans des identifications du magicien avec des entités surnaturelles ou dans les identifications du rôle des divinités invoquées. Ce qui frappe, c'est la concentration de ce type dans quelques sections isolées du papyrus magique de Londres et Leyde. Ainsi, on trouve la formation link $p 3 l \mathrm{~A}$ en 1,$12 ; 1,13 ; 1,21 ; 1,28$, contre un seul exemple de $i n k \mathrm{~A}(1,25)$ dans une formule de récitation. De telles observations donnent l'impression qu'il s'agit moins d'une distinction globale de deux constructions à signification différente, mais plutôt de prédilections personnelles qui se juxtaposent à cause du caractère de compilation du manuscrit.

\section{CONCLUSIONS}

L'étude des constructions discutées ici a servi à mettre en lumière le caractère spécifique du démotique tardif. Jusqu'ici, on ne l'a pas toujours considéré comme une entité en soi. Il a été assimilé à des stades bien plus anciens pour constituer "le démotique", ou bien il a été compris comme une sorte de copte en écriture démotique. En fait, il n'en est rien. Il faut bien admettre qu'il constitue un stade important de la langue égyptienne. Il affiche effectivement des développements qui mènent au copte, mais, dans bien des cas, il préserve encore systématiquement des constructions, qui ne seront plus utilisables en copte, ou bien les nouvelles formes n'ont pas encore acquis toute l'étendue qu'elles auront plus tard. Bien sûr, déjà le démotique archaïque et ptolémaïque contiennent bon nombre de constructions qui sont conservées sans grand changement jusqu'au copte, et le démotique tardif a fait quelques pas ultérieurs dans les régions qui séparent encore le démotique classique du copte. Ainsi, il est vraiment en route vers le copte, mais constitue aussi un état passager en soi.

Finalement, on doit noter qu'il ne sera plus possible de considérer le papyrus magique de Londres et Leyde comme une unité homogène représentant le démotique tardif. De nombreuses sections de charmes magiques spécifiques sont de provenance diverse et se distinguent dans l'usage de la langue. La grammaire du démotique profitera d'une plus grande sensibilité à l'égard de la divergence entre la date des manuscrits et la date de la composition - et aussi concernant la possibilité de rajeunissements partiels. 